\title{
Prevalence of Staphylococcus aureus and MRSA among Medical students: a
}

\section{literature review}

Prevalência de Staphylococcus aureus e MRSA entre estudantes de Medicina: uma revisão de

\section{literatura}

Prevalencia de Staphylococcus aureus y MRSA en estudiantes de Medicina: una revision de la

\section{literatura}

\author{
Amanda de Souza Lemos \\ ORCID: https://orcid.org/0000-0002-8837-2823 \\ State University of Ponta Grossa, Brazil \\ E-mail: amanda_soule@hotmail.com \\ Ana Carolina Mello Fontoura de Souza \\ ORCID: https://orcid.org/0000-0003-2324-1301 \\ State University of Ponta Grossa, Brazil \\ E-mail:acmfs00@gmail.com \\ Bruna Karas \\ ORCID: https://orcid.org/0000-0003-1301-9256 \\ State University of Ponta Grossa, Brazil \\ E-mail: brunakaras3@gmail.com \\ Camilla Mattia Calixto \\ ORCID: https://orcid.org/0000-0001-5464-0837 \\ State University of Ponta Grossa, Brazil \\ E-mail: calixtocamilla@gmail.com \\ Celine Iris Meijerink \\ ORCID: https://orcid.org/0000-0002-3964-5338 \\ State University of Ponta Grossa, Brazil \\ E-mail: celineirismeijerink@gmail.com \\ Felipe Câncio Nascimento \\ ORCID: https://orcid.org/0000-0003-4142-8946 \\ State University of Ponta Grossa, Brazil \\ E-mail: felipe_nascimento2.0@ hotmail.com \\ Gabriela Pires Corrêa Pinto \\ ORCID: https://orcid.org/0000-0003-4471-0613 \\ State University of Ponta Grossa, Brazil \\ E-mail: gabipires0702@gmail.com \\ Julia Henneberg Hessman \\ ORCID: https://orcid.org/0000-0002-9310-4508 \\ State University of Ponta Grossa, Brazil \\ E-mail: julia.hessman@gmail.com \\ Laura Bazzi Longo \\ ORCID: https://orcid.org/0000-0003-1396-1524 \\ State University of Ponta Grossa, Brazil \\ E-mail: laurabazzilongo@hotmail.com \\ José Carlos Rebuglio Vellosa \\ ORCID: https://orcid.org/0000-0003-4747-9197 \\ State University of Ponta Grossa, Brazil \\ E-mail: vellosajcr@hotmail.com \\ Elisangela Gueiber Montes \\ ORCID: https://orcid.org/0000-0003-1694-085X \\ State University of Ponta Grossa, Brazil \\ E-mail: elisangela.gueiber@uol.com.br
}

\begin{abstract}
Objective: The aim was to analyze the results of studies about the prevalence of Staphylococcus aureus and methicillin-resistant $S$. aureus among medical students. Methodology: A literature review was carried out from August to November 2020, being selected 19 articles from the Pubmed and "Biblioteca Virtual de Saúde" databases, using the descriptors "Methicillin-Resistant Staphylococcus aureus" and "Students, Medical". Studies from the last 10 years that target medical students and samples collected by nasal swab were included. Results: Several studies have
\end{abstract}


confirmed the hypothesis that, as students advanced in the academic years and, consequently, raised their exposure to hospital environments, colonization by methicillin-sensitive and resistant Staphylococcus aureus increased. However, some studies were divergent, not finding significant values for this association. The prevalences found also varied according to the place and country surveyed. Conclusion: In general, the greater the exposure to hospital environments, the higher the rate of colonization of students by methicillin-resistant Staphylococcus aureus and Staphylococcus aureus.

Keywords: Staphylococcus aureus; Methicillin-resistant Staphylococcus aureus; MRSA; Medical students; Clinical stage; Nasal carriage; Prevalence.

\begin{abstract}
Resumo
Objetivo: Analisar resultados de estudos com relação à prevalência de Staphylococcus aureus e Staphylococcus aureus meticilino-resistente entre os estudantes de medicina. Metodologia: Foi realizada uma revisão de literatura, de agosto a novembro de 2020, com a seleção de 19 artigos das bases de dados Pubmed e Biblioteca Virtual em Saúde (BVS), através dos descritores "Methicillin-Resistant Staphylococcus aureus" e "Students, Medical". Foram incluídos trabalhos dos últimos 10 anos que tivessem como população alvo estudantes de medicina e amostras coletadas por swab nasal. Resultados: Vários estudos comprovaram a hipótese de que, conforme os estudantes avançaram nos anos do curso e, consequentemente, ampliaram sua exposição a ambientes hospitalares, a colonização por Staphylococcus aureus sensível e resistente à meticilina aumentou. Entretanto, algumas pesquisas que divergem das primeiras, não encontraram valores significativos para essa associação. As prevalências encontradas variaram, também, conforme o local e país pesquisado. Conclusão: De modo geral, quanto maior foi a exposição a ambientes hospitalares, maior foi a taxa de colonização dos alunos por Staphylococcus aureus meticilino-resistente e Staphylococcus aureus.
\end{abstract}

Palavras-chave: Staphylococcus aureus; Staphylococcus aureus Resistente à Meticilina; Estudantes de medicina; Estágio clínico; Prevalência.

\title{
Resumen
}

Objetivo: Analizar los resultados de los estudios sobre la prevalencia de Staphylococcus aureus y Staphylococcus aureus resistente a la meticilina entre los estudiantes de medicina. Metodología: Se realizó una revisión bibliográfica, de agosto a noviembre de 2020, con la selección de 19 artículos de las bases de datos Pubmed y Biblioteca Virtual de Salud (BVS), utilizando los descriptores "Methicillin-Resistant Staphylococcus aureus" y "Students, Medical". Se incluyeron los artículos de los últimos 10 años que tenían como población objetivo a los estudiantes de medicina y a las muestras recogidas mediante hisopo nasal. Resultados: Varios estudios han apoyado la hipótesis de que, a medida que los estudiantes avanzaban en sus estudios de medicina y, en consecuencia, ampliaban su exposición a entornos hospitalarios, aumentaba la colonización por Staphylococcus aureus sensible a la meticilina y resistente a la meticilina. Sin embargo, algunos estudios que difieren de los anteriores no encontraron valores significativos para esta asociación. La prevalencia encontrada también varió según el lugar y el país encuestado. Conclusión: En general, cuanto mayor era la exposición al entorno hospitalario, mayor era la tasa de colonización de los estudiantes por Staphylococcus aureus resistente a la meticilina y Staphylococcus aureus.

Palabras clave: Staphylococcus aureus; Staphylococcus aureus Meticilin-Resistant; Estudantes de medicina; Estágio clínico; Prevalência.

\section{Introduction}

Nowadays, Staphylococcus aureus (S. aureus) is the leading cause of community and nosocomial infections (Okamo et al., 2016). This bacterium is an important colonizer of the humans' skin and mucosa, mainly anterior nasal cavity, but also perineum, pharynx, gastrointestinal tract, vagina and armpits (López-Aguilera et al., 2013). From the microbiota, in susceptible patients, the bacteria can infect skin, soft tissues, lungs, bones, joints and blood circulation (Chamberlain \& Singh, 2011). Thus, that is a significant cause of mortality and morbidity in immunosuppressed patients, patients on hemodialysis, with prolonged hospitalizations and advanced age, besides smoking, alcoholism and chronic respiratory diseases (Ma et al., 2011; Rampal et al., 2020). In 2017, the microorganism was included in the World Health Organization's (WHO) List of Priority Pathogens, ratifying its relevance on the world stage (Szymanek-Majchrzak et al., 2019).

In the 1960s, a strain of methicillin-resistant Staphylococcus aureus (MRSA) was first isolated (Jayaweera et al., 2020). It is now known that this variant is due to the $\operatorname{mec}(\mathrm{A})$ and SCCmec genes, which encode altered penicillin-binding proteins (PBP), conferring resistance to beta-lactams and methicillin (Alzoubi et al., 2020). With the emergence of this resistant strain, there has been an increase in the therapeutic complexity of hospital-acquired infections, as well as 
hospitalization time and treatment costs (Alzoubi et al., 2020; Rodríguez-Avial et al., 2013). Studies proves that individuals exposed to a hospital environment for long periods have a greater chance of carrying S. aureus or MRSA in the nasal microbiota and, consequently, healthcare workers can be a source of transmission of nosocomial infections (Alzoubi et al., 2020; Okamo et al., 2016). Most of the population is an intermediate carrier of S. aureus, while the rest is divided between those who are always colonized and those who never acquire the microorganism (Ansari et al., 2016). In 2011, MRSA infections were estimated to account for about 50-63\% of all nosocomial infections in the United States (Chamberlain \& Singh, 2011). The current percentage of MRSA among S. aureus has been increasing, however, worldwide, it varies by region, corresponding to rates of 20 to $80 \%$ of all S. aureus (Alzoubi et al., 2020). In South America, North America, Malta and Asia, MRSA prevalence rates were greater than 50\%. In South America, Brazil was one of the countries that showed prevalence greater than 50\% (Santos, 2018). However, despite being a microorganism of global relevance, in Brazil, there has been a scarcity of published data on the topic, which hinders the delineation of its real epidemiology in the country (Evangelista \& Oliveira, 2015). The variation in prevalence among countries can be explained by geographic location, the extent of environmental exposure to risk factors, as well as different policies for infection control and prevention (Okamo et al., 2016).

Several studies have investigated the prevalence of MSSA (methicillin-susceptible Staphylococcus aureus) and MRSA among medical students, in order to compare whether those on clinical postings, with greater contact in hospital institutions, had a higher prevalence of these microorganisms than students in the basic cycle, or only to analyze the profile of the students as carriers of the pathogens mentioned above. This review aims to make an analysis and interpretation of these studies, presenting a synthesis of the found data, besides discussing the reasons that led to the results presented by each one of them. Considering the small number of data found in the literature, this study aims to perform a literature review on the prevalence of MSSA and MRSA among medical students in different locations worldwide. In order to allow an epidemiological analysis and to direct prevention and treatment policies for these infections, as well as to encourage the inclusion of medical students in these actions.

\section{Methodology}

In this article, a literature review was conducted from August to November of 2020, using articles from the last 10 years for its writing. We included articles in Portuguese, English and Spanish that had medical students as target population and samples collected by nasal swab.

Initially, articles from the Scielo, Pubmed and "Biblioteca Virtual de Saúde" (BVS) databases were searched, applying the descriptors "Methicillin-Resistant Staphylococcus aureus" and "Students, Medical". From this search, four, 86 and 65 results were found, respectively, from which, one, 19 and 8 articles were selected (Table 1). 
Table 1 - Selection of the articles.

\begin{tabular}{|c|c|c|c|c|c|c|}
\hline Database & Descriptors & $\begin{array}{l}\text { Number } \\
\text { of results }\end{array}$ & $\begin{array}{l}\text { Number of } \\
\text { selected } \\
\text { articles }\end{array}$ & $\begin{array}{c}\text { Articles } \\
\text { Excluded After } \\
\text { Reading }\end{array}$ & $\begin{array}{l}\text { Remained in } \\
\text { the study }\end{array}$ & Selection date \\
\hline Scielo & $\begin{array}{c}\text { Methicillin- } \\
\text { Resistant } \\
\text { Staphylococcus } \\
\text { aureus AND } \\
\text { Students, Medical }\end{array}$ & 4 & 1 & 1 & $\overline{0}$ & $09 / 08 / 2020$ \\
\hline Pubmed & $\begin{array}{c}\text { Methicillin- } \\
\text { Resistant } \\
\text { Staphylococcus } \\
\text { aureus AND } \\
\text { Students, Medical }\end{array}$ & 86 & 19 & 2 & 17 & 09/08/2020 \\
\hline $\begin{array}{l}\text { Biblioteca Virtual de } \\
\text { Saúde (BVS) }\end{array}$ & $\begin{array}{c}\text { Methicillin- } \\
\text { Resistant } \\
\text { Staphylococcus } \\
\text { aureus AND } \\
\text { Students, Medical }\end{array}$ & 65 & 8 & 6 & 2 & $02 / 10 / 2020$ \\
\hline
\end{tabular}

Source: Authors.

From this initial selection, articles that involved a sample with graduate professionals, students from other courses (Chinese medicine, nursing, pharmacy and dentistry, medical labor sciences), non-nasal collections, or those involving the general population were excluded. The only exception was the article by Shadi A. Zakai (2015), which despite containing both resident physicians and medical students in its sample, made it possible to analyze the students in isolation, allowing the exclusion of only the part corresponding to resident physicians without having to eliminate the article as a whole. Thus, the final selection did not include articles from the Scielo database and included 17 articles from Pubmed and two from BVS, totaling 19 articles for the study.

After the criterion selection of articles, the studies were read and analyzed for data collection, evaluating the type of study, location, sample details, culture media and identification tests that were used, and the described resistance standard. Of these selected studies, most have a cross-sectional methodology and are concentrated in Asia, and none of them were conducted in Brazil.

Furthermore, in the selected papers, the prevalence of MRSA and S. aureus among medical students was emphasized. Therefore, the main objective of the present paper is to analyze the relation between the presence of MRSA and S. aureus and the time of clinical exposure, comparing the findings in preclinical and clinical students, as well as observing other risk factors that were presented in the studies.

\section{Results and Discussion}

The articles analyzed in this study varied both in location, being distributed in several countries, and in the culture media used for growth of the microorganism and analysis of the resistance profile. In addition, the samples collected were also varied. In general, we can divide medical students into those who were in the preclinical stage at the university at the time of sample collection and those who were already in clinical stage, the latter being in greater contact with the hospital and, therefore, presenting more risk of being carriers of S. aureus and MRSA, since one of the most common routes of infection is the health professional-patient route (Chamberlain \& Singh, 2011).

Regarding the culture media that were used, there was a prevalence in the use of sheep blood agar and salt mannitol agar for the cultivation of $S$. aureus, as well as Mueller Hinton agar to perform the antibiogram. In addition to these agars, 
some studies used methods diverging from each other, as in the study by Szymanek-Majchrzak et al. (2019) which used Chapman agar; the article by Ma et al. (2011), which used Columbia agar; and the work by Ansari et al. (2016), which applied DNAse agar. The studies by Jayaweera, Pilapitiya and Kumbukgolla (2020); Treesirichod, Hantagool and Prommalikit (2014); and Gualdoni et al. (2012), used MacConkey agar, with the latter two also making use of chocolate agar. The study by Rodríguez-Avial et al. (2013), on the other hand, used chromID SAID agar and chromID MRSA agar with methicillin for the growth of $S$. aureus and for the antibiogram, the diffusion method on MH2 agar was used.

Some articles also used nutrient broth for bacterial growth, such as brain heart infusion agar with the presence of $\mathrm{NaCl}$ at 7,5\%, tryptone soy broth enriched agar and mannitol salt agar, quoted sequentially as Jayaweera et al. (2020), LópezAguilera et al. (2013), Ho, Lai and Chow (2015), and Budri et al. (2018). The methodology presented in each article is resumed in Table 2.

Table 2 - Description of the methodology of each study.

\begin{tabular}{|c|c|c|c|c|}
\hline Study & Study period & Location & Identification tests of $S$. aureus & $\begin{array}{c}\text { Guidelines for diagnosis } \\
\text { of MRSA }\end{array}$ \\
\hline $\begin{array}{l}\text { Al-Tamimi et } \\
\text { al. }(2018)\end{array}$ & $2015-2016$ & $\begin{array}{l}\text { Hashemite } \\
\text { University }\end{array}$ & $\begin{array}{l}\text { Colony morphology (fermentation on MSA, beta } \\
\text { hemolysis on blood agar), Gram staining and } \\
\text { coagulase and catalase tests; } \\
\text { automatic Vitek } 2 \text { compact system; } \\
\text { slide latex agglutination test (for the detection of } \\
\text { PBP2); PCR. }\end{array}$ & $\begin{array}{c}\text { CLSI } \\
\text { (disc diffusion method) }\end{array}$ \\
\hline $\begin{array}{l}\text { Alzoubi et al. } \\
\quad(2020)\end{array}$ & $2018-2019$ & University of Jordan & $\begin{array}{c}\text { Colony morphology, Gram staining, fermentation } \\
\text { of glucose and mannitol, catalase and tube } \\
\text { coagulase test. }\end{array}$ & $\begin{array}{l}\text { CLSI (cefoxitin disc in } \\
\text { Mueller-Hinton agar) and } \\
\text { EUCAST (Kirby Bauer's } \\
\text { disc diffusion method) }\end{array}$ \\
\hline $\begin{array}{l}\text { Ansari et al. } \\
\quad(2016)\end{array}$ & March, 2014 & $\begin{array}{l}\text { Chitwan Medical } \\
\text { College }\end{array}$ & $\begin{array}{l}\text { Sheep blood agar, DNase agar and MSA plates. } \\
\text { Standard microbiological methods recommended } \\
\text { by American Society for Microbiology. }\end{array}$ & $\begin{array}{l}\text { CLSI (modified Kirby- } \\
\text { Bauer disk diffusion } \\
\text { method) }\end{array}$ \\
\hline $\begin{array}{l}\text { Budri et al. } \\
\quad(2018)\end{array}$ & $2014-2016$ & $\begin{array}{l}\text { Royal College of } \\
\text { Surgeons }\end{array}$ & $\begin{array}{c}\text { Colony morphology on SaSelect agar. MALDI } \\
\text { Biotyper. }\end{array}$ & $\begin{array}{c}\text { EUCAST } \\
\text { (fusidic acid MICs) }\end{array}$ \\
\hline $\begin{array}{l}\text { Chamberlain } \\
\text { and Singh } \\
(2011)\end{array}$ & 2011 & $\begin{array}{l}\text { Kirksville College } \\
\text { of Ostheopathic } \\
\text { Medicine }\end{array}$ & $\begin{array}{c}\text { Fermentation on MSA, catalase and coagulase } \\
\text { test, } \\
\text { PCR for the sigA and rsbU genes. }\end{array}$ & NS \\
\hline $\begin{array}{l}\text { Chen } \text { et al. } \\
\quad \text { (2012) }\end{array}$ & 2009 & $\begin{array}{l}\text { Chang Gung } \\
\text { University }\end{array}$ & Beta hemolysis on blood agar and coagulase test. & $\begin{array}{c}\text { CLSI } \\
\text { (disc diffusion method) }\end{array}$ \\
\hline Efa et al. (2019) & $\begin{array}{c}\text { May to } \\
\text { August } 2016\end{array}$ & $\begin{array}{l}\text { Jimma University } \\
\text { Medical Center }\end{array}$ & $\begin{array}{c}\text { Fermentation on MSA, Gram staining, catalase } \\
\text { and coagulase tests. }\end{array}$ & $\begin{array}{c}\text { CLSI } \\
\text { (disc diffusion method) }\end{array}$ \\
\hline $\begin{array}{l}\text { Geliebter } \text { et al. } \\
\quad(2020)\end{array}$ & $\begin{array}{l}\text { January to } \\
\text { June } 2018\end{array}$ & $\begin{array}{l}\text { University of } \\
\text { Cartagena }\end{array}$ & $\begin{array}{c}\text { Colony morphology, Gram stain, catalase test, } \\
\text { tube coagulase test, and latex agglutination test. } \\
\text { Genomic DNA; PCR-amplification using specific } \\
\text { primers for nuc and mecA. }\end{array}$ & CLSI \\
\hline $\begin{array}{l}\text { Gualdoni et } \\
\text { al.(2012) }\end{array}$ & NS & $\begin{array}{l}\text { Medical University } \\
\text { of Vienna }\end{array}$ & $\begin{array}{l}\text { Characteristic phenotypically growth on blood } \\
\text { agar, Gram staining, catalase test. } \\
\text { Fermentation on MSA, analytical Profile Index } \\
\text { and PCR. }\end{array}$ & $\begin{array}{l}\text { CLSI } \\
\text { (microtiter dilution } \\
\text { method) }\end{array}$ \\
\hline Ho et al. (2015) & $\begin{array}{l}2006-2013 \\
(\text { except } 2008)\end{array}$ & $\begin{array}{l}\text { University of Hong } \\
\text { Kong }\end{array}$ & $\begin{array}{l}\text { Colony morphology, Gram staining, latex } \\
\text { agglutination, tube coagulase, mannitol, ornithine } \\
\text { and DNase tests. } \\
\text { spa and SCCmec typing; PCR for PVL genes. }\end{array}$ & $\begin{array}{c}\text { CLSI } \\
\text { (disc diffusion method) }\end{array}$ \\
\hline
\end{tabular}




\begin{tabular}{|c|c|c|c|c|}
\hline $\begin{array}{l}\text { Jayaweera et al. } \\
\quad(2020)\end{array}$ & $2015-2017$ & $\begin{array}{l}\text { Rajarata University } \\
\text { of Sri Lanka }\end{array}$ & $\begin{array}{c}\text { Fermentation on MSA; DNAse and tube } \\
\text { coagulase tests. }\end{array}$ & $\begin{array}{l}\text { CLSI } \\
\text { (disc diffusion and } \\
\text { oxacillin agar plate } \\
\text { dilution method) }\end{array}$ \\
\hline $\begin{array}{l}\text { López- Aguilera } \\
\text { et al. (2013) }\end{array}$ & 2012 & $\begin{array}{l}\text { Hospital } \\
\text { Universitario } 12 \text { de } \\
\text { Octubre }\end{array}$ & $\begin{array}{l}\text { Culture on MSA; PCR; D-test; electrophoresis; } \\
\text { typification of multilocus sequences. }\end{array}$ & $\begin{array}{c}\text { CLSI } \\
\text { (disc diffusion method) }\end{array}$ \\
\hline Ma et al. (2011) & $2008-2009$ & $\begin{array}{l}\text { China Medical } \\
\text { University }\end{array}$ & Gram staining, catalase and coagulase tests. & $\begin{array}{c}\text { CLSI } \\
\text { (macrodilution method) }\end{array}$ \\
\hline $\begin{array}{l}\text { Orlin et al. } \\
\quad \text { (2017) }\end{array}$ & $2012-2014$ & $\begin{array}{l}\text { Galilee of Bar-Ilan } \\
\text { University }\end{array}$ & $\begin{array}{l}\text { Culture and colony isolation on a selective } \\
\text { chromogenic growth medium; } \\
\text { Gram staining, catalase test, and rapid } \\
\text { agglutination test; PCR. }\end{array}$ & $\begin{array}{c}\text { CLSI } \\
\text { (disc diffusion method on } \\
\text { Muller-Hinton agar) }\end{array}$ \\
\hline $\begin{array}{l}\text { Rodríguez- } \\
\text { Avial } \text { et al. } \\
\quad(2013)\end{array}$ & $2008-2012$ & $\begin{array}{l}\text { Universidad } \\
\text { Complutense de } \\
\text { Madrid }\end{array}$ & Culture in selective and chromogenic medium. & $\begin{array}{c}\text { CLSI } \\
\text { (disc diffusion method) }\end{array}$ \\
\hline $\begin{array}{l}\text { Syafinaz et al. } \\
\quad(2012)\end{array}$ & $\begin{array}{l}\text { January to } \\
\text { June } 2011\end{array}$ & $\begin{array}{l}\text { University Putra } \\
\text { Malaysia }\end{array}$ & $\begin{array}{c}\text { Culture on MSA, Gram staining and coagulase } \\
\text { test. }\end{array}$ & $\begin{array}{c}\text { CLSI } \\
\text { (disc diffusion method) }\end{array}$ \\
\hline $\begin{array}{l}\text { Szymanek- } \\
\text { Majchrzak et al. } \\
\text { (2019) }\end{array}$ & $2014-2016$ & $\begin{array}{l}\text { Medical University } \\
\text { of Warsaw }\end{array}$ & $\begin{array}{l}\text { Pastorex-Staph-Plus (BioRad) and/or the VITEK- } \\
\text { MS system (Biomerieux). } \\
\text { Presence of the mecA/mecC and mupA genes } \\
\text { detected with PCR technique. }\end{array}$ & $\begin{array}{l}\text { EUCAST (disk diffusion } \\
\text { and/or broth } \\
\text { microdilution method) }\end{array}$ \\
\hline $\begin{array}{l}\text { Treesirichod et } \\
\quad \text { al. }(2014)\end{array}$ & 2012 & $\begin{array}{l}\text { Srinakharinwirot } \\
\text { University }\end{array}$ & $\begin{array}{l}\text { Colony morphology on culture plates; Gram } \\
\text { staining, catalase production and tube coagulase } \\
\text { tests. }\end{array}$ & $\begin{array}{c}\text { CLSI } \\
\text { (Kirby Bauer's disc } \\
\text { diffusion method) }\end{array}$ \\
\hline Zakai (2015) & $2014-2015$ & $\begin{array}{l}\text { King Abdulaziz } \\
\text { University }\end{array}$ & $\begin{array}{c}\text { Fermentation on MSA, Gram staining, catalase } \\
\text { and coagulase tests; } \\
\text { PCR. }\end{array}$ & $\begin{array}{c}\text { CLSI } \\
\text { (disc diffusion method) }\end{array}$ \\
\hline
\end{tabular}

Source: Authors. (CLSI: Clinical \& Laboratory Standards Institute; EUCAST: European Committee on Antimicrobial Susceptibility Testing; MICs: Minimum Inibitory Concentrations; MSA: Mannitol Salt Agar; NS: not specified; PCR: polymerase chain reaction).

The articles' samples were classified in preclinical or clinical according to the definition presented in each research, since this data can vary because of the curriculum of each university. In studies where this definition was not available, clinical students were considered the ones from the third year and forward. The results of each study were detailed in Table 3, in which initially can be observed a large variability showed in the results, considering that good part can be explained by the prevalence difference of the microorganism in each country, also by the different pathogen control actions and due the irregular exposures to environmental factors in each institution (Okamo et al., 2016). 
Table 3 - Description of the results of each study.

\begin{tabular}{|c|c|c|c|c|c|}
\hline Study & Study design & Region & $\begin{array}{l}\text { Prevalence in } \\
\text { preclinical } \\
\text { students }\end{array}$ & $\begin{array}{l}\text { Prevalence in } \\
\text { clinical students }\end{array}$ & Total prevalence \\
\hline $\begin{array}{l}\text { Al-Tamimi et } \\
\text { al. }(2018)\end{array}$ & $\begin{array}{l}\text { Cross- } \\
\text { sectional }\end{array}$ & Jordan & $\begin{array}{c}189 \text { students } \\
\left(1^{\text {st }}, 2^{\text {nd }} \text { and } 3^{\text {rd }}\right. \\
\text { years }) \\
\text { NS }\end{array}$ & $\begin{array}{l}101 \text { students } \\
\left(4^{\text {th }} \text { year }\right) \\
\text { NS }\end{array}$ & $\begin{array}{c}290 \text { students } \\
\text { Traditional methods: } \\
\text { SA: } 66(22.7 \%) \\
\text { MRSA: } 12(4.1 \%) \\
\text { Molecular tests and Vitek 2: } \\
\text { SA: } 63(21.7 \%) \\
\text { MRSA: } 7(2.4 \%)\end{array}$ \\
\hline $\begin{array}{l}\text { Alzoubi et al. } \\
(2020)\end{array}$ & $\begin{array}{c}\text { Cross- } \\
\text { sectional }\end{array}$ & Jordan & $\begin{array}{c}105 \text { students } \\
\text { SA: } 9(8.6 \%) \\
\text { MRSA: } 2(1.9 \%) \\
\end{array}$ & $\begin{array}{c}105 \text { students } \\
\text { SA: } 15(14.2 \%) \\
\text { MRSA: } 3(2.8 \%)\end{array}$ & $\begin{array}{c}210 \text { students } \\
\text { SA: } 24(11.4 \%) \\
\text { MRSA: } 5(2.4 \%)\end{array}$ \\
\hline $\begin{array}{l}\text { Ansari et al. } \\
\text { (2016) }\end{array}$ & $\begin{array}{c}\text { Cross- } \\
\text { sectional }\end{array}$ & $\begin{array}{l}\text { Bharatpur, } \\
\text { Nepal }\end{array}$ & $\begin{array}{c}200 \text { students } \\
\left(1^{\text {st }} \text { year }\right) \\
\text { SA: } 30(15 \%) \\
\text { MRSA: } 8(4 \%) \\
\end{array}$ & - & $\begin{array}{c}200 \text { preclinical students. } \\
\text { SA: } 30(15 \%) \\
\text { MRSA: } 8(4 \%)\end{array}$ \\
\hline $\begin{array}{l}\text { Budri et al. } \\
\text { (2018) }\end{array}$ & $\begin{array}{l}\text { Cross- } \\
\text { sectional }\end{array}$ & Ireland & $\begin{array}{c}444 \text { students } \\
\text { SA: } 137(31 \%) \\
\text { MRSA: } 9(2 \%)\end{array}$ & - & $\begin{array}{c}444 \text { preclinical students } \\
\text { SA: } 137(31 \%) \\
\text { MRSA: } 9(2.02 \%)\end{array}$ \\
\hline $\begin{array}{l}\text { Chamberlain } \\
\text { and Singh } \\
(2011)\end{array}$ & $\begin{array}{c}\text { Cross- } \\
\text { sectional }\end{array}$ & $\begin{array}{l}\text { Kirksville, } \\
\text { USA }\end{array}$ & $\begin{array}{c}132 \text { students } \\
\left(2^{\text {nd }} \text { year }\right) \\
\text { SA: } 62(47 \%) \\
\text { MRSA: } 2(1.5 \%)\end{array}$ & - & $\begin{array}{c}132 \text { preclinical students. } \\
\text { SA: } 62(47 \%) \\
\text { MRSA: } 2(1.5 \%)\end{array}$ \\
\hline $\begin{array}{l}\text { Chen et al. } \\
\text { (2012) }\end{array}$ & $\begin{array}{c}\text { Cross- } \\
\text { sectional }\end{array}$ & $\begin{array}{l}\text { Taoyuan, } \\
\text { Taiwan }\end{array}$ & $\begin{array}{c}167 \text { students } \\
\text { SA: } 28(16.8 \%) \\
\text { MRSA: } 4(2.4 \%)\end{array}$ & $\begin{array}{c}155 \text { students } \\
\text { SA: } 34(21.9 \%) \\
\text { MRSA: } 3(1.9 \%)\end{array}$ & $\begin{array}{c}322 \text { students } \\
\text { SA: } 62(19.25 \%) \\
\text { MRSA: } 7(2.17 \%)\end{array}$ \\
\hline $\begin{array}{l}\text { Efa et al. } \\
(2019)\end{array}$ & $\begin{array}{c}\text { Cross- } \\
\text { sectional }\end{array}$ & $\begin{array}{l}\text { Jimma, } \\
\text { Ethiopia }\end{array}$ & - & $\begin{array}{c}4^{\text {th }} \text { year: } \\
166 \text { students } \\
\text { SA: } 28(16.9 \%) \\
\text { MRSA: } 6(3.6 \%) \\
5^{\text {th }} \text { year: } \\
125 \text { students } \\
\text { SA: } 23(18.4 \%) \\
\text { MRSA: } 9(7.2 \%) \\
6^{\text {th }} \text { year: } \\
\text { 80 students } \\
\text { SA: } 31(38.8 \%) \\
\text { MRSA: } 16(20 \%)\end{array}$ & $\begin{array}{l}371 \text { clinical students } \\
\text { SA: } 82(22.1 \%) \\
\text { MRSA: } 31(8.4 \%)\end{array}$ \\
\hline $\begin{array}{l}\text { Geliebter } \text { et } \\
\text { al. }(2020)\end{array}$ & $\begin{array}{c}\text { Cross- } \\
\text { sectional }\end{array}$ & $\begin{array}{l}\text { Cartagena, } \\
\text { Colombia }\end{array}$ & $\begin{array}{c}143 \text { students } \\
\left.\text { ( } 2^{\text {nd }} \text { year }\right) \\
\text { SA: } 40(28 \%) \\
\text { MRSA: } 12(8.4 \%)\end{array}$ & - & $\begin{array}{c}143 \text { preclinical students } \\
\text { SA: } 40(28 \%) \\
\text { MRSA: } 12(8.4 \%)\end{array}$ \\
\hline $\begin{array}{l}\text { Gualdoni et } \\
\text { al. (2012) }\end{array}$ & $\begin{array}{c}\text { Cross- } \\
\text { sectional }\end{array}$ & Austria & - & $\begin{array}{c}79 \text { students } \\
\text { SA: } 20(25.31 \%) \\
\text { MRSA: } 0 \\
\end{array}$ & $\begin{array}{c}79 \text { clinical students. } \\
\text { SA: } 20(25.31 \%) \\
\text { MRSA: } 0 \\
\end{array}$ \\
\hline $\begin{array}{l}\text { Ho et al. } \\
\text { (2015) }\end{array}$ & $\begin{array}{l}\text { Cross- } \\
\text { sectional }\end{array}$ & Hong Kong & $\begin{array}{c}1149 \text { students } \\
\left(1^{\text {st }} \text { year }\right) \\
\text { SA: } 532(46.3 \%) \\
\text { MRSA: } 6(0.5 \%)\end{array}$ & - & $\begin{array}{c}1149 \text { preclinical students } \\
\text { SA: } 532(46.3 \%) \\
\text { MRSA: } 6(0.5 \%)\end{array}$ \\
\hline $\begin{array}{l}\text { Jayaweera } \text { et } \\
\text { al. }(2020)\end{array}$ & $\begin{array}{l}\text { Cross- } \\
\text { sectional with } \\
\text { follow-up } \\
\text { approach }\end{array}$ & Sri Lanka & $\begin{array}{c}110 \text { students } \\
1^{\text {st }} \text { year: } \\
\text { MRSA: } 7(6.4 \%) \\
\text { After } 6 \text { months: } \\
\text { MRSA: } 31(28.1 \%)\end{array}$ & $\begin{array}{c}3^{\text {rd }} \text { year: } \\
\text { 114 students } \\
\text { MRSA: } 29(25.4 \%) \\
5^{\text {th }} \text { year: } \\
\text { 119 students } \\
\text { MRSA: } 59 \\
(49.57 \%)\end{array}$ & $\begin{array}{c}343 \text { students } \\
\text { SA: NS } \\
\text { MRSA: } 7(6.36 \%) \text { in } \\
\text { preclinical, } 29(25.4 \%) \text { in } 3^{\text {rd }} \\
\text { year and } 59(49.7 \%) \text { in } 5^{\text {th }} \text { year. }\end{array}$ \\
\hline
\end{tabular}




\begin{tabular}{|c|c|c|c|c|c|}
\hline $\begin{array}{l}\text { López- } \\
\text { Aguilera et al. } \\
\text { (2013) }\end{array}$ & $\begin{array}{c}\text { Cross- } \\
\text { sectional }\end{array}$ & $\begin{array}{l}\text { Madrid, } \\
\text { Spain }\end{array}$ & $\begin{array}{c}70 \text { students } \\
\left.\text { ( } 3^{\text {rd }} \text { year }\right) \\
\text { SA: } 24(34.3 \%) \\
\text { MRSA: } 0\end{array}$ & $\begin{array}{c}70 \text { students } \\
\left(6^{\text {th }} \text { year }\right) \\
\text { SA: } 31(44.3 \%) \\
\text { MRSA: } 3(4.3 \%)\end{array}$ & $\begin{array}{c}140 \text { students } \\
\text { SA: } 55(39.3 \%) \\
\text { MRSA: } 3(2.1 \%)\end{array}$ \\
\hline $\begin{array}{l}\text { Ma et al. } \\
\text { (2011) }\end{array}$ & $\begin{array}{l}\text { Cross- } \\
\text { sectional }\end{array}$ & $\begin{array}{c}\text { Shenyang, } \\
\text { China }\end{array}$ & $\begin{array}{c}2103 \text { students } \\
\text { SA: } 234(11,13 \%) \\
\text { MRSA: } 22(1 \%)\end{array}$ & - & $\begin{array}{l}2103 \text { preclinical students } \\
\text { SA: } 234(11,13 \%) \\
\text { MRSA: } 22(1,05 \%)\end{array}$ \\
\hline $\begin{array}{l}\text { Orlin et al. } \\
\text { (2017) }\end{array}$ & Longitudinal & $\begin{array}{l}\text { Safed, } \\
\text { Israel }\end{array}$ & $\begin{array}{c}58 \text { students } \\
\text { Sample 1: } \\
\text { SA: } 19(33 \%) \\
\text { MRSA: } 4(7 \%)\end{array}$ & $\begin{array}{c}58 \text { students } \\
\text { Sample } 2-13 \\
\text { months: } \\
\text { SA: } 22(38 \%) \\
\text { MRSA: } 6(10 \%) \\
\text { Sample } 3-19 \\
\text { months: } \\
\text { SA: } 24(41 \%) \\
\text { MRSA: } 3(5 \%)\end{array}$ & $\begin{array}{c}58 \text { students (three samples from } \\
\text { each student) } \\
\text { SA: } 32 \text { students }(55 \%) \\
\text { MRSA: } 7 \text { students }(12 \%)\end{array}$ \\
\hline $\begin{array}{l}\text { Rodríguez- } \\
\text { Avial et al. } \\
(2013)\end{array}$ & Longitudinal & $\begin{array}{c}\text { Madrid, } \\
\text { Spain }\end{array}$ & $\begin{array}{c}52 \text { students } \\
\left(3^{\text {rd }} \text { year }\right) \\
\text { SA: } 14(26.92 \%) \\
\text { MRSA: } 0 \\
\end{array}$ & $\begin{array}{c}81 \text { students } \\
\left(6^{\text {th }} \text { year }\right) \\
\text { SA: } 37(46.25 \%) \\
\text { MRSA: } 1(1.25 \%)\end{array}$ & $\begin{array}{c}81 \text { students from the same } \\
\text { class. } \\
\text { SA: } 37(46.25 \%) \\
\text { MRSA: } 1(1.25 \%)\end{array}$ \\
\hline $\begin{array}{l}\text { Syafinaz et al. } \\
(2012)\end{array}$ & $\begin{array}{c}\text { Cross- } \\
\text { sectional }\end{array}$ & $\begin{array}{l}\text { Selangor, } \\
\text { Malaysia }\end{array}$ & $\begin{array}{c}111 \text { students } \\
\left(1^{\text {st }} \text { and } 2^{\text {nd }}\right) \\
\text { SA: } 55(49.5 \%) \\
\text { MRSA: } 0 \\
\end{array}$ & $\begin{array}{c}98 \text { students } \\
\left(3^{\text {rd }}, 4^{\text {th }} \text { and } 5^{\text {th }}\right) \\
\text { SA: } 7(7.1 \%) \\
\text { MRSA: } 0 \\
\end{array}$ & $\begin{array}{l}209 \text { students } \\
\text { SA: } 62(29.6 \%) \\
\text { MRSA: } 0\end{array}$ \\
\hline $\begin{array}{l}\text { Szymanek- } \\
\text { Majchrzak et } \\
\text { al. }(2019)\end{array}$ & Longitudinal & $\begin{array}{l}\text { Warsaw, } \\
\text { Poland }\end{array}$ & $\begin{array}{c}955 \text { students } \\
\left(3^{\text {rd }} \text { year }\right) \\
\text { SA: } 245(25.7 \%) \\
\text { MRSA: } 1(0.1 \%) \\
\end{array}$ & - & $\begin{array}{c}955 \text { preclinical students } \\
\text { SA: } 245(25.7 \%) \\
\text { MRSA: } 1(0.1 \%)\end{array}$ \\
\hline $\begin{array}{l}\text { Treesirichod } \\
\text { et al. (2014) }\end{array}$ & $\begin{array}{l}\text { Longitudinal } \\
\text { follow-up }\end{array}$ & $\begin{array}{l}\text { Nakhon } \\
\text { Nayok } \\
\text { Province, } \\
\text { Thailand }\end{array}$ & $\begin{array}{c}128 \text { students } \\
\text { Sample } 1- \\
\text { prior to the clinical } \\
\text { rotations: } \\
\text { SA: } 38(29.7 \%) \\
\text { MRSA: } 0\end{array}$ & $\begin{array}{c}128 \text { students } \\
\text { Sample } 2- \\
\text { after the first ward } \\
\text { rotation: } \\
\text { SA: } 39(30.5 \%) \\
\text { MRSA: } 0 \\
\text { Sample } 3- \\
\text { at the end of the } \\
\text { rotation schedule: } \\
\text { SA: } 50(39.4 \%) \\
\text { MRSA: } 0 \\
\end{array}$ & $\begin{array}{l}128 \text { students (three samples } \\
\text { from each student) } \\
\text { SA: } 25(20.3 \%) \text { - persistent } \\
\text { carrier } \\
\text { MRSA: } 0\end{array}$ \\
\hline Zakai (2015) & $\begin{array}{l}\text { Cross- } \\
\text { sectional }\end{array}$ & $\begin{array}{l}\text { Jeddah, } \\
\text { Saudi } \\
\text { Arabia }\end{array}$ & $\begin{array}{l}32 \text { control samples } \\
\text { from } 3^{\text {rd }} \text { year } \\
\text { students } \\
\text { SA: } 100 \%\end{array}$ & $\begin{array}{c}150 \text { students } \\
\left(6^{\text {th }} \text { year and }\right. \\
\text { internship }) \\
\text { SA: } 38(25.3 \%) \\
\text { MRSA: } 10(6.7 \%)\end{array}$ & $\begin{array}{l}150 \text { clinical students } \\
\text { SA: } 38(25.3 \%) \\
\text { MRSA: } 10(6.7 \%)\end{array}$ \\
\hline
\end{tabular}

Source: Authors. (NS: not specified; SA: Staphylococcus aureus)

Hospital exposure presents itself as an important risk factor for the colonization by $S$. aureus and MRSA, an example of that is evident in the study conducted by Rodríguez-Avial et al. (2013), whereupon students from the same class were monitored, first when they were in the third year and again when they reached the sixth year, after having been exposed to activities in ambulatories and hospitals for a longer time. In the first analysis, 14 of 52 students were colonized with $S$. aureus $(26,92 \%)$ and none of them had presented MRSA. Therefore, in the collection after three years, 81 students participated and of these, $37(46,25 \%)$ had S. aureus and $1(1,25 \%)$ were colonized with MRSA.

In the Efa et al. (2019) studies, samples taken from students of those same undergraduate years were analyzed, all from clinical cycle, and the same increasing pattern was observed in the prevalence of individuals with MRSA (fourth year: 3.6\%; fifth year: 7.2\%; and sixth year: $20 \%$ ), as well as S. aureus in general. In the studies realized in Thailand conducted by Treesirichod et al. $(2013,2014)$, third year students were analyzed in three different moments, the first before they started the 
wards rotations, the other after the first rotation and the last collection at the end of all rotations. Among the 128 students, although there were none colonized by MRSA, it was possible to observe a gradual increase in the prevalence of MSSA, which went from $29,7 \%$ in the first collection to $39,4 \%$ in the third.

Likewise, other studies have also shown a significantly association between MRSA transport and time of exposure to the hospital. In Jayaweera et al. (2020), three batches of medical students, from first, third and fifth year, were screened for MRSA colonization. Before having been exposed to healthcare settings, only $6.4 \%$ of $1^{\text {st }}$ year students were MRSA carriers. After six months of starting their clinical training, these same students were re-screened, and the MRSA colonization was increased up to $28.1 \%$. The $5^{\text {th }}$ year students, who were exposed to healthcare settings for two years and six months duration, showed an even greater increase with $49.6 \%$ MRSA colonization.

The Graphic 1 illustrates how most of articles that studied both preclinical and clinical students found an increase in the prevalence of $S$. aureus as the hospital exposure of students rises over the course.

Graph 1 - Comparison between the prevalence of Staphylococcus aureus in the studies that investigated samples in clinical and preclinical students.

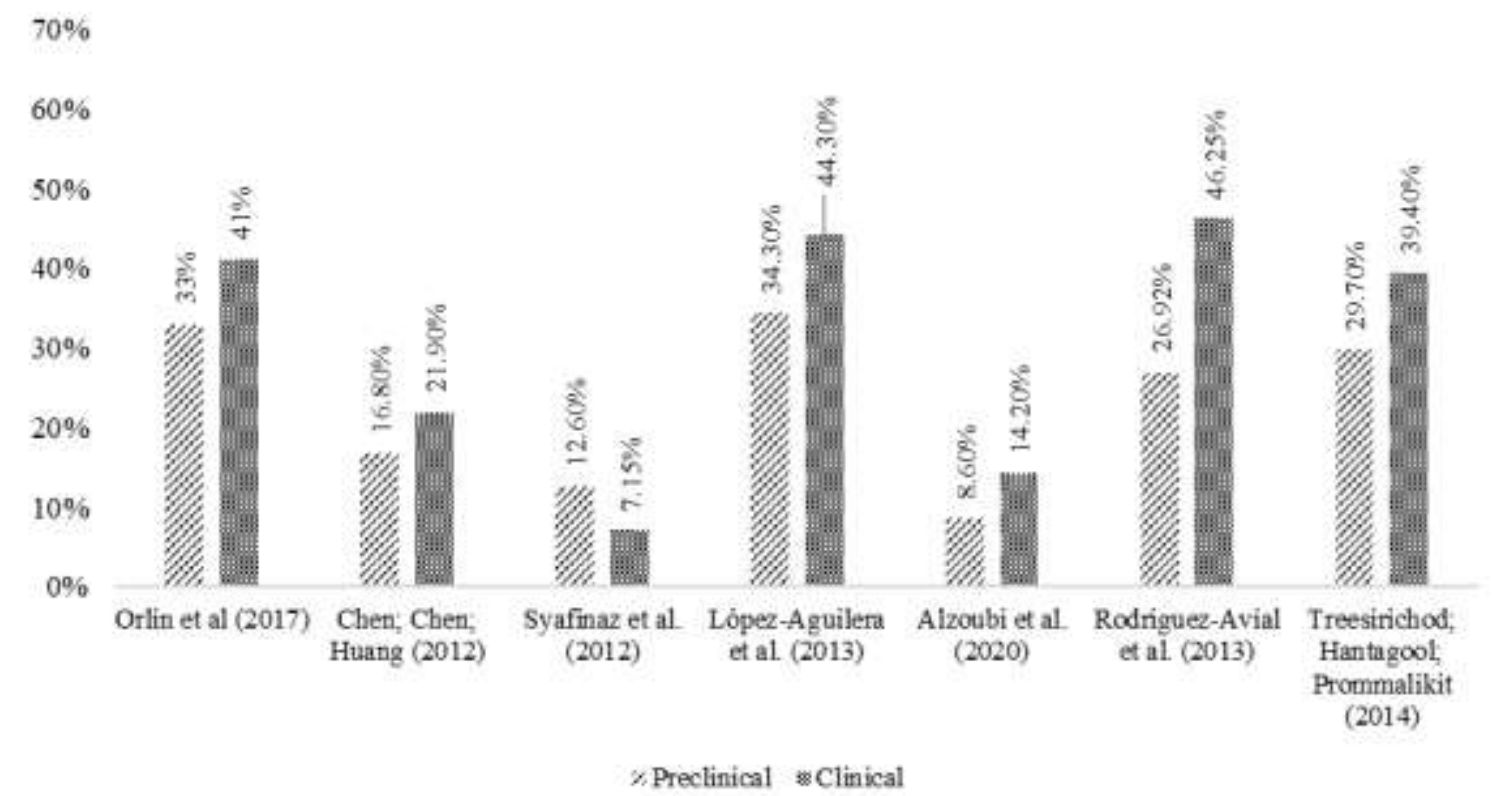

Note: to make the graphs, the percentages were calculated using the number of samples informed in the studies in cases where the values presented were only absolute. Source: Authors.

However, some cross-sectional studies highlighted that, in their institutions, there was no significance between hospital exposure and the presence of MRSA. According to Chen et al. (2012), the prevalence of MRSA in preclinicals was $2.4 \%$ while clinical was $1.9 \%$ after 1 to 2 years of exposure. In the study by Alzoubi et al. (2020), MRSA was found in 1.9\% of students in preclinical years and 2.8\% among clinical years, but the years of each cycle were not distinguished. Whereas in two studies, no MRSA cases were found (Gualdoni et al., 2012; Syafinaz et al., 2012). The results found in other studies, in addition to those described in Table 3, have been summarized in Graphs 2 and 3. 
Graph 2 - Prevalence of Staphylococcus aureus in each study.

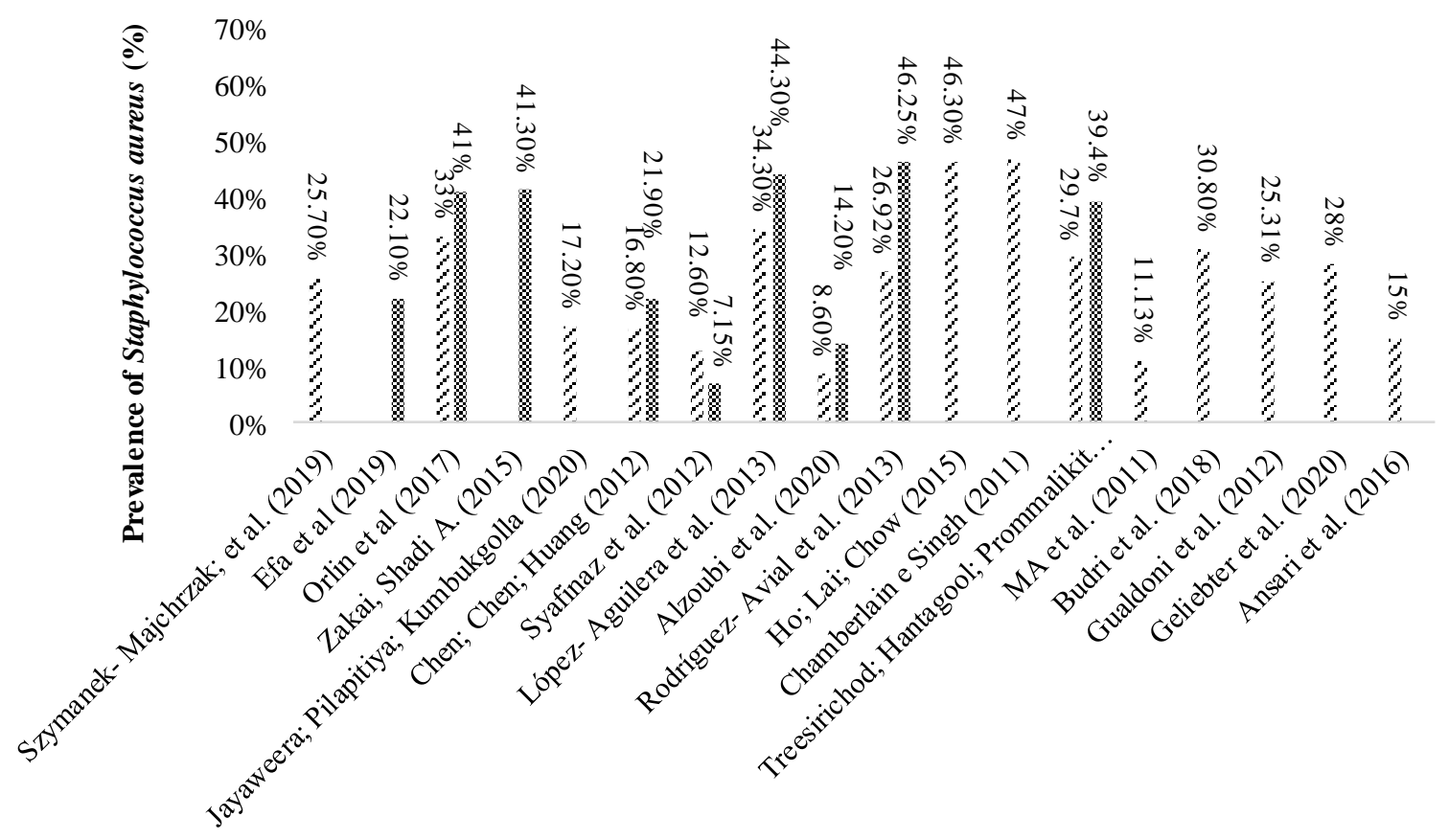

Study

${ }^{\circ}$ Preclinical ${ }^{\otimes}$ Clinical

Note: to make the graphs, the percentages were calculated using the number of samples informed in the studies in cases where the values presented were only absolute. Source: Authors.

Graph 3 - Prevalence of methicillin-resistant Staphylococcus aureus in each study.

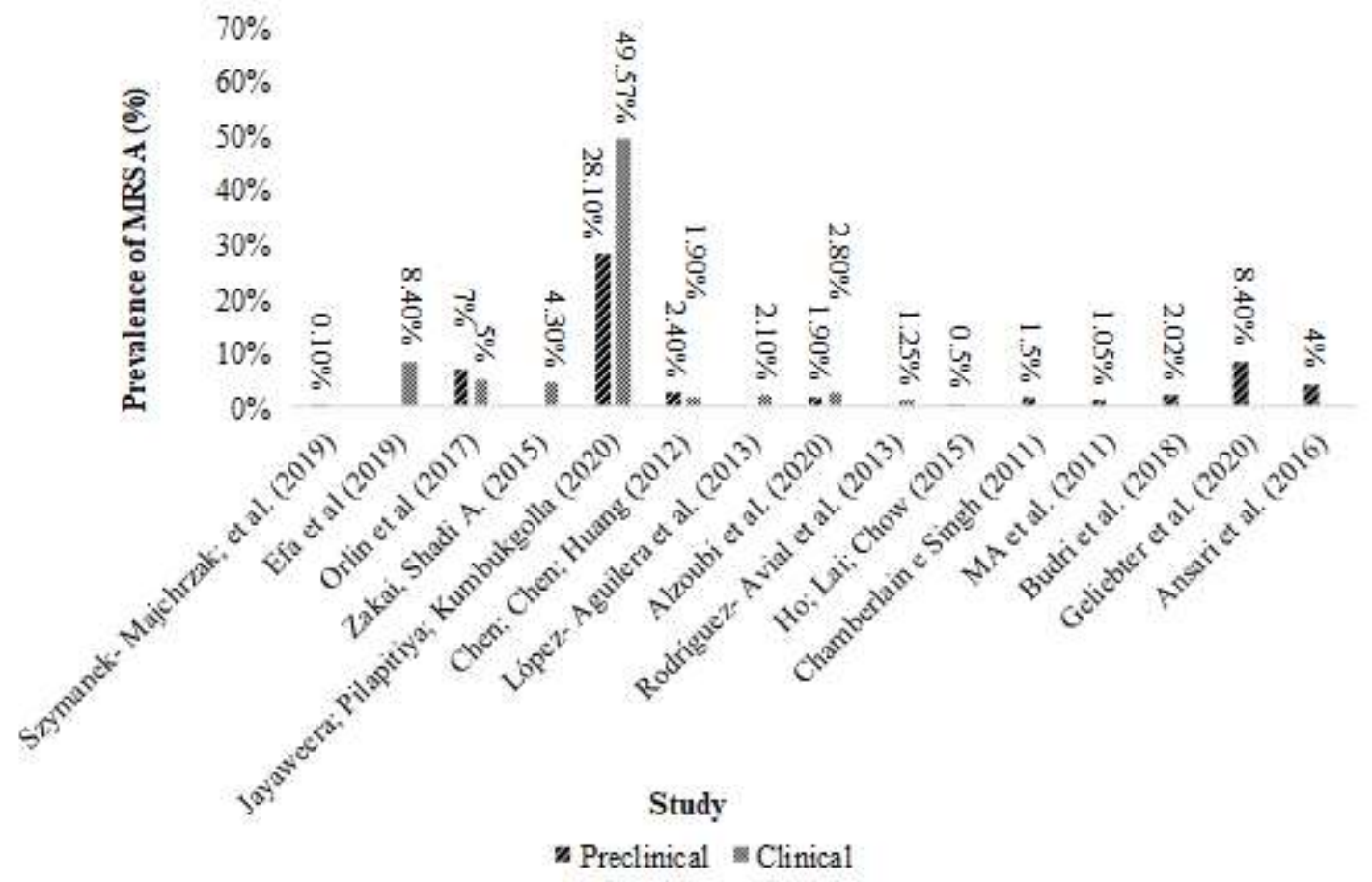

Note: to make the graphs, the percentages were calculated using the number of samples informed in the studies in cases where the values presented were only absolute. MRSA: methicillin-resistant Staphylococcus aureus. Source: Authors. 
In some studies, risk factors assessed through questionnaires were also described. The trial Ma et al. (2011) reports a statistically significant association between the carriage of $S$. aureus and chronic sinusitis $(\mathrm{OR}=2.06,95 \% \mathrm{CI}$ : $1.34-3.18)$, smoking $(\mathrm{OR}=1.63,95 \% \mathrm{CI}: 1.02$ - 2.61) and frequent contact with hospitals (OR = 1.65, 95\% CI: 1.23 - 2.23). Meanwhile, the authors of the study Chen et al. (2012) indicated as risk factors for carrying S. aureus: being male $(\mathrm{OR}=2.098,95 \%$ CI,1.039 - $4.235 \mathrm{p}=0.036)$ and age 23 years or older $(\mathrm{OR}=2.121,95 \% \mathrm{CI}, 1.194-3.768, \mathrm{p}=0.009)$. According to Ansari et al. (2016), there was a statistically significant relationship between colonization of $S$. aureus in the nasal microbiota and the presence of upper respiratory tract infections $(\mathrm{p}=0.035)$, in addition to mentioning the absence of a relationship with risk factors declared as usual (hypertension, kidney disease, lower respiratory tract infection, gastrointestinal disease, hospital admission, hospital visit and medication use, these last three factors considering the last trimester). Consistent with the others, the article by Al-Tamimi et al. (2018) demonstrated that nasal colonization by $S$. aureus showed a significant correlation with male gender $(\mathrm{p}=0.049)$ and with the presence of chronic diseases $(\mathrm{p}=0.006)$.

On the other hand, the Gualdoni et al. (2012) study reports that there was no statistically significant association between S. aureus colonization and clinical exposure, gender and smoking. Finally, there were divergences between the studies' results regarding antibiotic therapy, as the article by Ma et al. (2011) study considered antibiotic use in the past three months (OR $=1.45,95 \%$ CI: 1.01 - 2.08) as a risk factor for S. aureus carriage, while Ansari et al. (2016) reported finding no correlation between antibiotic therapy in the last trimester, whereas the Chen et al. (2012) study stated that the sample that used antibiotics in the last year had a significantly lower rate of S. aureus carriage than the one that did not (17.1\% vs. $25 \%$, p $=0.028)$.

\section{Conclusion}

After a thorough analysis of the selected articles, it was possible to conclude that the increase of hospital exposure time, i.e., the increase of time spent in outpatient clinics and wards by students as they progress through the medical course (preclinical to clinical), increases the possibility of contamination by Staphylococcus aureus and MRSA, since the studies that pointed otherwise were not significant.

This exposure, besides representing risk to the students themselves, also contributes to the transmission of this pathogen to the patients who seek care in hospital environments, which can worsen the prognosis, increase mortality rates and length of hospital stay. Another point to be considered is that the presence of other factors may also be related to the portability of $S$. aureus, such as chronic sinusitis, smoking, male gender, individuals older than 23 years old, other upper respiratory tract infections and the presence of chronic diseases. In general, the use of antibiotic therapy in the last 3 months had divergences regarding its influence on the carriage of $S$. aureus.

Therefore, it is clear the need for structuring educational actions, both in hospitals and in medical teaching institutions, aimed at hand washing practices, monitoring of hospital infections and awareness of professionals and students about these issues. Since the data studied in this paper vary according to the time and places studied, it is recommended that new studies on the subject be carried out constantly, in order to evaluate the evolution of the profile of MRSA and S. aureus infections among medical students.

\section{References}

Al-Tamimi, M., Himsawi, N., Abu-Raideh, J., Jazar, D. A., Al-Jawaldeh, H., Mahmoud, S. A. H., Hijjawi, N., \& Hawamdeh, H. (2018). Nasal colonization by methicillin-sensitive and methicillin-resistant staphylococcus aureus among medical students. Journal of Infection in Developing Countries, 12(5), 326-335. https://doi.org/10.3855/jidc. 9908

Alzoubi, H., Al Madadha, M., Al-Mnayyis, A., Azzam, M., Aldawoud, A., Hwaiti, D., Tarbiah, M., Abu Ajamieh, M., \& Qatamin, M. (2020). Detection of Methicillin Susceptible and Resistant Staphylococcus aureus Nasal Carriage and Its Antibiotic Sensitivity among Basic and Clinical Years Medical Students. 
Healthcare, 8(2), 161. https://doi.org/10.3390/healthcare8020161

Ansari, S., Gautam, R., Shrestha, S., Ansari, S. R., Subedi, S. N., \& Chhetri, M. R. (2016). Risk factors assessment for nasal colonization of Staphylococcus aureus and its methicillin resistant strains among pre-clinical medical students of Nepal. BMC Research Notes, 9(1), 1-8. https://doi.org/10.1186/s13104-0162021-7

Budri, P. E., Shore, A. C., Coleman, D. C., Kinnevey, P. M., Humpreys, H., \& Fitzgerald-Hughes, D. (2018). Observational cross-sectional study of nasal staphylococcal species of medical students of diverse geographical origin, prior to healthcare exposure: Prevalence of SCCmec, fusC, fusB and the arginine catabolite mobile element (ACME) in the absence of sele. BMJ Open, 8(4). https://doi.org/10.1136/bmjopen-2017-020391

Chamberlain, N. R., \& Singh, V. K. (2011). Prevalence of methicillin resistant Staphylococcus aureus in nasal samples from preclinical second-year medical students. Missouri Medicine, 108(5), 373-376.

Chen, C. Y. C. S., Chen, C. Y. C. S., \& Huang, Y. C. (2012). Nasal carriage rate and molecular epidemiology of methicillin-resistant Staphylococcus aureus among medical students at a Taiwanese university. International Journal of Infectious Diseases, 16(11), e799-e803. https://doi.org/10.1016/j.ijid.2012.07.004

Efa, F., Alemu, Y., Beyene, G., Gudina, E. K., \& Kebede, W. (2019). Methicillin-resistant Staphylococcus aureus carriage among medical students of Jimma University, Southwest Ethiopia. Heliyon, 5(1), e01191. https://doi.org/10.1016/j.heliyon.2019.e01191

Evangelista, S. de S., \& Oliveira, A. C. de. (2015). Staphylococcus aureus meticilino resistente adquirido na comunidade: um problema mundial. Revista Brasileira de Enfermagem, 68(1), 136-143. https://doi.org/10.1590/0034-7167.2015680119p

Geliebter, J., Reyes, N., Montes, O., Figueroa, S., Tiwari, R., Sollecito, C. C., Emmerich, R., Usyk, M., \& Burk, R. D. (2020). Staphylococcus aureus nasal carriage and microbiome composition among medical students from Colombia: A cross-sectional study. F1000Research, 9(78), 1-16. https://doi.org/10.12688/f1000research.22035.2

Gualdoni, G. A., Lingscheid, T., Tobudic, S., \& Burgmann, H. (2012). Low nasal carriage of drug-resistant bacteria among medical students in Vienna. GMS Krankenhaushygiene Interdisziplinär, 7(1), 1-4. https://doi.org/10.3205/dgkh000188

Ho, P. L., Lai, E. L., \& Chow, K. H. (2015). Carriage of meticillin-susceptible and -resistant Staphylococcus aureus by medical students in Hong Kong. Journal of Hospital Infection, 91(2), 184-185. https://doi.org/10.1016/j.jhin.2015.06.012

Jayaweera, J. A. A. S., Pilapitiya, S., \& Kumbukgolla, W. (2020). The relationship between the exposure to healthcare settings and colonization with methicillin-resistant Staphylococcus aureus among medical students. Germs, 10(1), 34-43. https://doi.org/10.18683/germs.2020.1217

López-Aguilera, S., Del Mar Goñi-Yeste, M., Barrado, L., González-Rodríguez-Salinas, C. M., Otero, J. R., \& Chaves, F. (2013). Colonización nasal por Staphylococcus aureus en estudiantes de medicina: Importancia en la transmisión hospitalaria. Enfermedades Infecciosas y Microbiologia Clinica, 31(8), 500 505. https://doi.org/10.1016/j.eimc.2012.12.005

Ma, X. X., Sun, D. D., Wang, S., Wang, M. L., Li, M., Shang, H., Wang, E. H., \& Luo, E. J. (2011). Nasal carriage of methicillin-resistant Staphylococcus aureus among preclinical medical students: Epidemiologic and molecular characteristics of methicillin-resistant S. aureus clones. Diagnostic Microbiology and Infectious Disease, 70(1), 22-30. https://doi.org/10.1016/j.diagmicrobio.2010.12.004

Okamo, B., Moremi, N., Seni, J., Mirambo, M. M., Kidenya, B. R., \& Mshana, S. E. (2016). Prevalence and antimicrobial susceptibility profiles of Staphylococcus aureus nasal carriage among pre-clinical and clinical medical students in a Tanzanian University Microbiology. BMC Research Notes, 9(1), 16. https://doi.org/10.1186/s13104-016-1858-0

Orlin, I., Rokney, A., Onn, A., Glikman, D., \& Peretz, A. (2017). Hospital clones of methicillin-resistant Staphylococcus aureus are carried by medical students even before healthcare exposure. Antimicrobial Resistance and Infection Control, 6(1), 1-10. https://doi.org/10.1186/s13756-017-0175-2

Rampal, S., Zainuddin, N. H., Elias, N. A., Jamaluddin, T. Z. M. T., Maniam, S., Teh, S. W., \& Subbiah, S. K. (2020). Colonization of methicillin-resistant staphylococcus aureus (MRSA) among medical students in tertiary institution in Central Malaysia. Antibiotics, 9(7), 1-9. https://doi.org/10.3390/antibiotics9070382

Rodríguez-Avial, C., Álvarez-Novoa, A., Losa, A., \& Picazo, J. J. (2013). Aumento significativo de la colonización por Staphylococcus aureus entre los estudiantes de medicina durante la realización de las prácticas en el hospital. Enfermedades Infecciosas y Microbiologia Clinica, 31(8), 516-519. https://doi.org/10.1016/j.eimc.2012.09.017

Santos, C. R. L. (2018). Vigilância epidemiológica de Staphylococcus aureus resistente a meticilina isolados de um hospital terciário público de Dourados/MS [Universidade Federal da Grande Dourados]. http://repositorio.ufgd.edu.br/jspui/handle/prefix/1072

Syafinaz, A. N., Nur Ain, N. Z. im, Nadzirah, S. N., Fatimah, J. S., Shahram, A., \& Nasir, M. D. M. (2012). Staphylococcus aureus nasal carriers among medical students in A medical school. Medical Journal of Malaysia, 67(6), 636-638.

Szymanek-Majchrzak, K., Kosiński, J., Żak, K., Sułek, K., Młynarczyk, A., \& Młynarczyk, G. (2019). Prevalence of methicillin resistant and mupirocinresistant Staphylococcus aureus strains among medical students of Medical University in Warsaw. Przeglad Epidemiologiczny, 73(1), 39-48. https://doi.org/10.32394/pe.73.05

Treesirichod, A., Hantagool, S., \& Prommalikit, O. (2013). Nasal carriage and antimicrobial susceptibility of Staphylococcus aureus among medical students at the HRH Princess Maha Chakri Sirindhorn Medical Center, Thailand: A cross sectional study. Journal of Infection and Public Health, 6(3), 196-201. https://doi.org/10.1016/j.jiph.2012.12.004

Treesirichod, A., Hantagool, S., \& Prommalikit, O. (2014). Nasal carriage and antimicrobial susceptibility of Staphylococcus aureus among medical students at the HRH Princess Maha Chakri Sirindhorn Medical Center, Thailand: A follow-up study. Journal of Infection and Public Health, 7(3), 205-209. https://doi.org/10.1016/j.jiph.2013.12.003

Zakai, S. A. (2015). Prevalence of methicillin-resistant staphylococcus aureus nasal colonization among medical students in Jeddah, Saudi Arabia. Saudi Medical Journal, 36(7), 807-812. https://doi.org/10.15537/smj.2015.7.11609 